\title{
Polypyrimidine tract binding protein and Notch1 are independently re-expressed in glioma
}

\author{
Hannah C Cheung ${ }^{1,2}$, Lynda J Corley ${ }^{3}$, Gregory N Fuller ${ }^{3}$, Ian E McCutcheon ${ }^{4}$ and \\ Gilbert J Cote ${ }^{1,2}$
}

\begin{abstract}
${ }^{1}$ Department of Endocrine Neoplasia and Hormonal Disorders, The University of Texas MD Anderson Cancer Center, Houston, TX, USA; ${ }^{2}$ The University of Texas Graduate School of Biomedical Sciences at Houston, Houston, TX, USA; ${ }^{3}$ Department of Pathology, The University of Texas MD Anderson Cancer Center, Houston, TX, USA and ${ }^{4}$ Department of Neurosurgery, The University of Texas MD Anderson Cancer Center, Houston, TX, USA
\end{abstract}

\begin{abstract}
Polypyrimidine tract binding protein (PTB) is expressed in developing mammalian astrocytes, absent in mature adult astrocytes, and aberrantly elevated in gliomas. It is unclear whether PTB is a coincidental marker of tumor progression or a significant mediator of tumorigenesis. In developing Drosophila, the absence of the PTB homolog, hephaestus, results in increased Notch activity. Since Notch is a well-known inducer of glial cell fate, we determined whether overexpression of PTB in glial cell tumors provides a selective growth advantage by inhibiting activated Notch (Notch1IC)-mediated differentiation. To do this, we performed an immunohistochemical analysis for expression of PTB, activated Notch1 (Notch1IC), Hes1 (a Notch target), and GFAP on an extensive human tissue microarray that included 246 gliomas, 10 gliosarcomas, and 10 normal brains. Statistically significant PTB overexpression was seen in all glioma grades, with the highest increase in grade IV tumors. Notch1IC was also abnormally expressed in gliomas except in a subset of grade IV tumors in which it was absent. This decrease in Notch1IC was not associated with increased PTB expression. We conclude that PTB, and Notch1 serve as independent and functionally unlinked markers of glioma progression.

Modern Pathology (2006) 19, 1034-1041. doi:10.1038/modpathol.3800635; published online 26 May 2006
\end{abstract}

Keywords: glioma; polypyrimidine tract binding protein; notch; tissue microarray

Multiple genetic aberrations are known to contribute to glioma formation, progression, invasion, and maintenance. Notable examples include epidermal growth factor receptor (EGFR) amplification, $\mathrm{LOH}$ 10, TP53 mutations, CDKN2A deletions, 1p loss, and PTEN mutations. ${ }^{1}$ Unfortunately, few of these genetic abnormalities have been found to be informative prognostic indicators of survival. Even in the largest population-based study to date, only LOH 10 was associated with shorter survival in patients with glioblastoma (GBM). ${ }^{2}$ As a result, there have been renewed efforts to find more clinically relevant glioma markers, especially those that would delineate the glioma cell of origin. Similarities between stem cells and tumor cells have led to the belief that tumors, including those of the brain,

Correspondence: Dr GJ Cote, PhD, Department of Endocrine Neoplasia and Hormonal Disorders, Unit 435, The University of Texas MD Anderson Cancer Center, 1515 Holcombe Blvd, Houston, TX 77030, USA.

E-mail: gcote@mdanderson.org

Received 15 February 2006; revised 24 April 2006; accepted 25 April 2006; published online 26 May 2006 evolve from stem cells. ${ }^{3-5}$ Subpopulations of glioma cells have been identified that express progenitor markers such as nestin or stem cell markers such as CD133 and like stem cells, they maintain their ability to self-renew., ${ }^{4,6}$ Furthermore, altered or aberrant signaling is frequently observed in key developmental or differentiation pathways involving EGFR, PTEN, transforming growth factor $\beta$ and Notch. ${ }^{5,8-10}$ Therefore, the identification of aberrant components of developmental pathways may reveal new prognostic factors and/or novel therapeutic targets.

Polypyrimidine tract binding protein (PTB) is a multifunctional component of mRNA metabolism that affects alternative splicing, $3^{\prime}$-end formation, polyadenylation, mRNA stability, internal ribosome entry site-independent translation, and possibly transcription. ${ }^{11,12}$ During fetal mouse brain development (E11.5-14.5), PTB levels are high in developing astrocytes. However, in the adult mouse brain, PTB is absent in mature astrocytes and occurs at low levels in neural progenitors of the choroid plexus and in the ependymal regions near the ventricles. ${ }^{13}$ In a similar manner, the adult human brain has little 
or no PTB expression in normal astrocytes and oligodendrocytes, with low expression in ependymocytes, the choroid plexus epithelium, microglia, the arachnoid, and the adenohypophysis. ${ }^{14}$ Resurgent expression of PTB has been observed in astrocyte-derived gliomas that is analogous to the expression of other tumor-associated developmental markers. ${ }^{14}$

PTB is a potential tumor marker, but the developmental and differentiation pathways it targets are unknown. In Drosophila, knockouts of the PTB homolog, hephaestus (heph), are lethal and various heph mutants have defects in spermatogenesis and wing formation. ${ }^{15,16}$ The first evidence that the heph and Notch developmental pathways are linked was in the heph genetic mosaics, in which heph-null clonal regions had increased Notch activity. Mature Notch is a heterodimer membrane receptor that plays a key role in several cellular differentiation pathways. In vertebrate neurogenesis, Notch dictates commitment of neural progenitors into the astrocytic lineage rather than the default neuronal lineage. In the astrocytic lineage, it directs terminal differentiation of precursors into astrocytes, as opposed to oligodendrocytes. ${ }^{17-19}$ Activation of Notch is mediated through the binding of membrane-bound ligands, Delta or Serrate/Jagged, located on neighboring cells. The act of ligand binding initiates dissociation of the receptor domains and triggers two cleavages: the extracellular segment by an ADAM metalloproteinase and the intracellular transmembrane by presenilin-dependent proteases. The final form of intracellular Notch (Notch1IC) translocates to the nucleus to activate expression of genes such as the hairy and enhancer of split 1 (HES1). Mammalian Notch1 has been implicated to act as either an oncogene in tumors such as T-cell leukemia and breast cancer or a tumor suppressor in tumors such as small cell lung and skin cancer. ${ }^{20-28}$ Increased Notch2 expression and signaling was also found in mouse models of medulloblastoma. Abrogating the Notch pathway in these models decreased tumor cell viability. ${ }^{29}$

The involvement of Notch in both neural development and cancer led us to investigate whether PTB could inhibit Notch activity in gliomas. We were further encouraged by recent results of depressed Notch1 levels in high-grade gliomas, which we hoped was due to enhanced PTB activity. ${ }^{9}$ Therefore, we used a large glioma tissue microarray to determine possible association between PTB and Notch1 in gliomagenesis.

\section{Materials and methods}

\section{Tissue Microarray}

A tissue microarray was constructed of 266 core biopsy samples, in duplicate of tumors derived from University of Texas-MD Anderson Cancer Center patients and normal brain tissues as controls (see
Table 1 A list of the core samples from the glioma tissue microarray

\begin{tabular}{lcc}
\hline Tissue type $^{\mathrm{a}}$ & $\begin{array}{c}\text { WHO } \\
\text { grade }\end{array}$ & $\begin{array}{c}\text { Number of } \\
\text { samples }\end{array}$ \\
\hline Low-grade astrocytoma (LGA) & II & 9 \\
Anaplastic astrocytoma (AA) & III & 48 \\
Glioblastoma (GBM) & IV & 70 \\
Gliosarcoma (GS) & IV & 10 \\
Oligodendroma (O) & II & 40 \\
Anaplastic oligodendroma (AO) & III & 40 \\
Mixed oligoastrocytoma (MOA) & II & 24 \\
Anaplastic mixed oligoastrocytoma (AMOA) & III & 15 \\
Normal brain (NB) & - & 10 \\
\hline
\end{tabular}

${ }^{\mathrm{a}}$ The 266 samples exist in duplicate and are arranged according to tissue type: astrocytomas (LGA, AA, GBM), oligodendromas (O, AO), mixed gliomas (MOA, AMOA), and normal brain (NB).

Table 1). Tumors were classified by one of us (GNF) into World Health Organization glioma histologic types and grades. Normal brain samples were derived surgical specimens of cerebral cortex and subjacent white matter resected to permit access to deep metastatic tumors. All samples were obtained under an approved Institution Review Board protocol.

\section{Immunohistochemical Analysis}

PTB immunohistochemistry was performed using a monoclonal anti-PTB antibody (DH3 clone). ${ }^{30} \mathrm{Un}$ purified culture medium derived from DH3 hybridoma cells was used as a source of antibody (a gift from Dr Susan Berget, Baylor College of Medicine). A $4-\mu$ m-thick section was deparaffinized in xylene for $5 \mathrm{~min}$ and rehydrated in graded ethanols to distilled water. Antigen retrieval was achieved with a rice steamer by placing the section in Dako target retrieval solution (citrate buffer, $\mathrm{pH}$ 6.0, Dako, Glostrup, Denmark) for $30 \mathrm{~min}$. The section was then blocked with $3 \%$ hydrogen peroxide/methanol for $15 \mathrm{~min}$ and incubated overnight at $4^{\circ} \mathrm{C}$ with antiPTB antibody (1:5 in Dako Antibody Diluent). After washing in phosphate-buffered saline (PBS), the section was incubated with Dako Mouse Envision Polymer and developed in DAB substrate for 4.5 min. Nuclei were counterstained with Mayer's hematoxylin.

Anti-Notch1IC antibody was obtained from Cell Signaling Technology, Inc. (Beverly, MA, USA), and anti-Hes1 was obtained from Chemicon International Inc. (Temecula, CA, USA). After deparaffinization and rehydration, antigen retrieval was performed by incubating sections in Tris-buffered saline with Tween-20, $\mathrm{pH} 7.6$, for $30 \mathrm{~min}$ in a rice steamer. Endogenous peroxidase activity was blocked with $3 \%$ hydrogen peroxide/methanol for $15 \mathrm{~min}$, followed by overnight incubation at $4^{\circ} \mathrm{C}$ with either anti-Notch1IC 1:100 in Dako Antibody Diluent containing $8 \%$ Power Block, BioGenex ${ }^{\mathrm{TM}}$, 
San Ramon, CA, USA) or anti-Hes1 (1:500 in Dako Antibody Diluent containing $8 \%$ Power Block) antibodies. After being washed in PBS, the slides were incubated with Dako Rabbit Envision Polymer for $30 \mathrm{~min}$ and developed in $\mathrm{DAB}\left(3^{\prime} 3^{\prime}\right.$ diaminobenzidine) substrate (Dako) for $2.5 \mathrm{~min}$. Nuclei were counterstained with Mayer's hematoxylin.

Mouse monoclonal anti-GFAP was purchased from BD Biosciences Pharmingen (San Diego, CA, USA). After deparaffinization and rehydration, the section was blocked with $3 \%$ hydrogen peroxide/ methanol for $5 \mathrm{~min}$ and rinsed three times in PBS. Antigen retrieval was performed in citrate buffer in a rice steamer for $45 \mathrm{~min}$. The slide was placed on a Dako Autostainer (DakoCytomation, Glostrup, Denmark) for addition of anti-GFAP (1:6000) for $30 \mathrm{~min}$. Dako LSAB2 kit components containing biotinylated anti-mouse IgG secondary antibody and enzyme-labelled strepavidin polymer were then added for $30 \mathrm{~min}$ each. The cores were washed and then developed in AEC (3-amino-9-ethylcarbazole) substrate for $16 \mathrm{~min}$ before removal from the stainer and counterstained with Mayer's hematoxylin.

\section{Immunohistochemical Staining Analysis}

The staining analysis was performed using the GenoMx Vision Automated Digital Imaging System (BioGenex, Inc., San Ramon, CA, USA). Each antibody-probed slide was scanned and the software was used to derive quantitative measures of tissue area, positive chromagen staining, and intensity of staining. To detect tissue area, parameters were set to recognize nonwhite regions within a standardized square containing each core. To measure positive chromagen staining, the color hue, saturation, and intensity parameters were set to optimally distinguish brown DAB or red AEC stained cells from the hematoxylin counterstain. For each antibody, these parameters were then stratified into three chromagen staining intensities (low, medium, and high). Therefore, each core sample had its own quantitative data set that defined: the total tissue area, the proportion of positively stained tissue area, and the percentage of low, medium, and high staining intensity within the positively stained area. For Figures 2 and 3, we used these values to score cores as 'Negative', 'Low Positive', or 'High Positive.' We considered staining to be positive when the total chromagen-stained area of the core was greater than $10 \%$ or on direct observation found highly localized staining to subcellular regions such as the nucleus. A core was scored as 'Low Positive' when greater than $50 \%$ of the stained area was low intensity. A core was scored as 'High Positive' when the percentage of low staining was less than $50 \%$, indicating that the majority of the staining was of medium and high intensity. All remaining cores were scored as 'Negative.' For Figure 4, the sum of the medium and high intensity values were used directly in order to provide a more quantitative comparison of staining. Multivariate $\chi^{2}$ tests were performed using the SPSS program, version 11 for Mac OS X (SPSS, Chicago, IL, USA). The Pearson correlation coefficient was used to determine $P$ values.

\section{Results}

\section{PTB and Activated Notch1 Levels were Significantly Higher in Gliomas}

To determine levels of PTB expression and Notch1 activity in gliomas, we performed immunohistochemical analyses using a glioma tissue microarray. The staining for each core was quantified using the GenoMx Vision Automated Digital Imaging System and scored as 'Negative,' 'Low Positive,' or 'High Positive', according to the criteria described in Materials and methods. Figure 1 shows representative staining and scoring of normal brain and GBM cores. The normal brain panels are all from white matter; there was no difference between white and gray matter staining. The results of the immunohistochemical analyses for each antibody are provided in Figure 2. The differences in sample number result from loss of patient cores during processing or removal because of staining artifacts. Where duplicate cores could be analyzed, there was scoring concordance of $85 \%$ for PTB and Notch1IC, 99\% for Hes1, and $82 \%$ for GFAP. Duplicate samples were randomized and combined with the remaining single cores to form the highest informative population for statistical evaluation.

There were no statistically significant differences in GFAP staining associated with tumor grade $(P=0.319)$. Positive staining for PTB was predominantly nuclear, with some cytoplasmic staining present in all samples (Figure 1). In normal brain tissue, PTB levels were scored as Negative. Examination of individual cores at higher magnification revealed occasional low-level staining for PTB in individual astrocytes, as we previously reported. ${ }^{14}$ In all tumor grades, most cores scored either Low or High Positive, with a significantly higher proportion of grade IV gliomas scoring High Positive than in grades II and III gliomas $(P<0.001)$. These results show aberrant overexpression of PTB in gliomas compared with normal brain $(P<0.001)$ (Figure 2).

In Drosophila, the loss of PTB is associated with activation of Notch. ${ }^{16}$ Therefore, we expected that the observed increases in PTB expression would be associated with a concomitant reduction in Notch1IC. However, the opposite outcome was observed (Figure 2). Only one sample of normal brain had Low Positive staining; the remainder $(89 \%)$ had no significant staining for Notch1IC. In the grades II and III gliomas, there was a significant increase in positive staining $(P<0.001)$ that was predominantly nuclear. However, a substantial 


\section{Normal Brain}

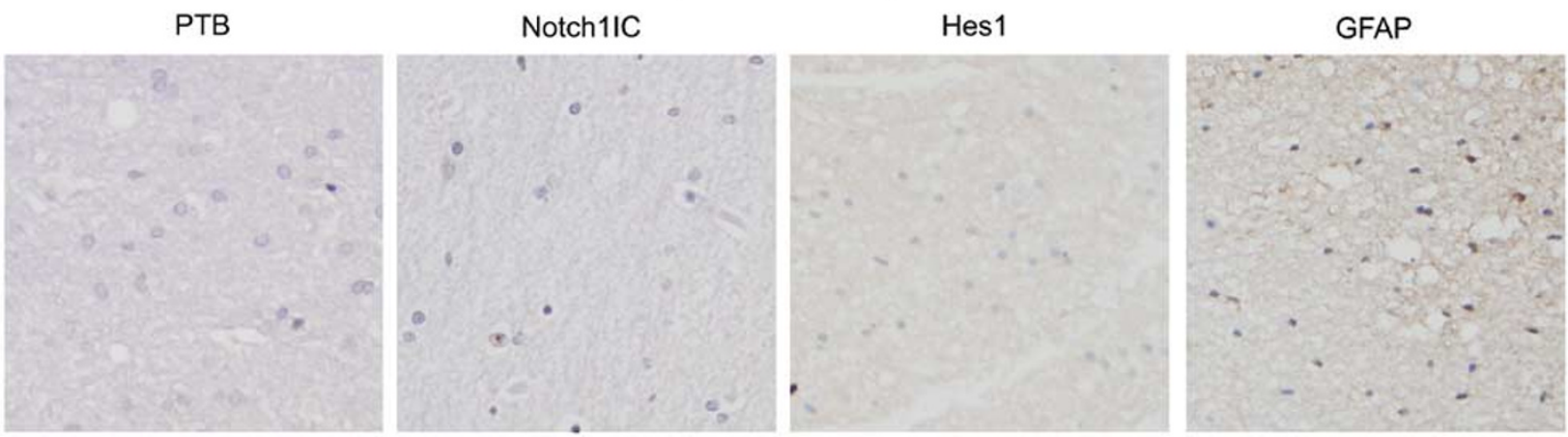

\section{Glioblastoma}
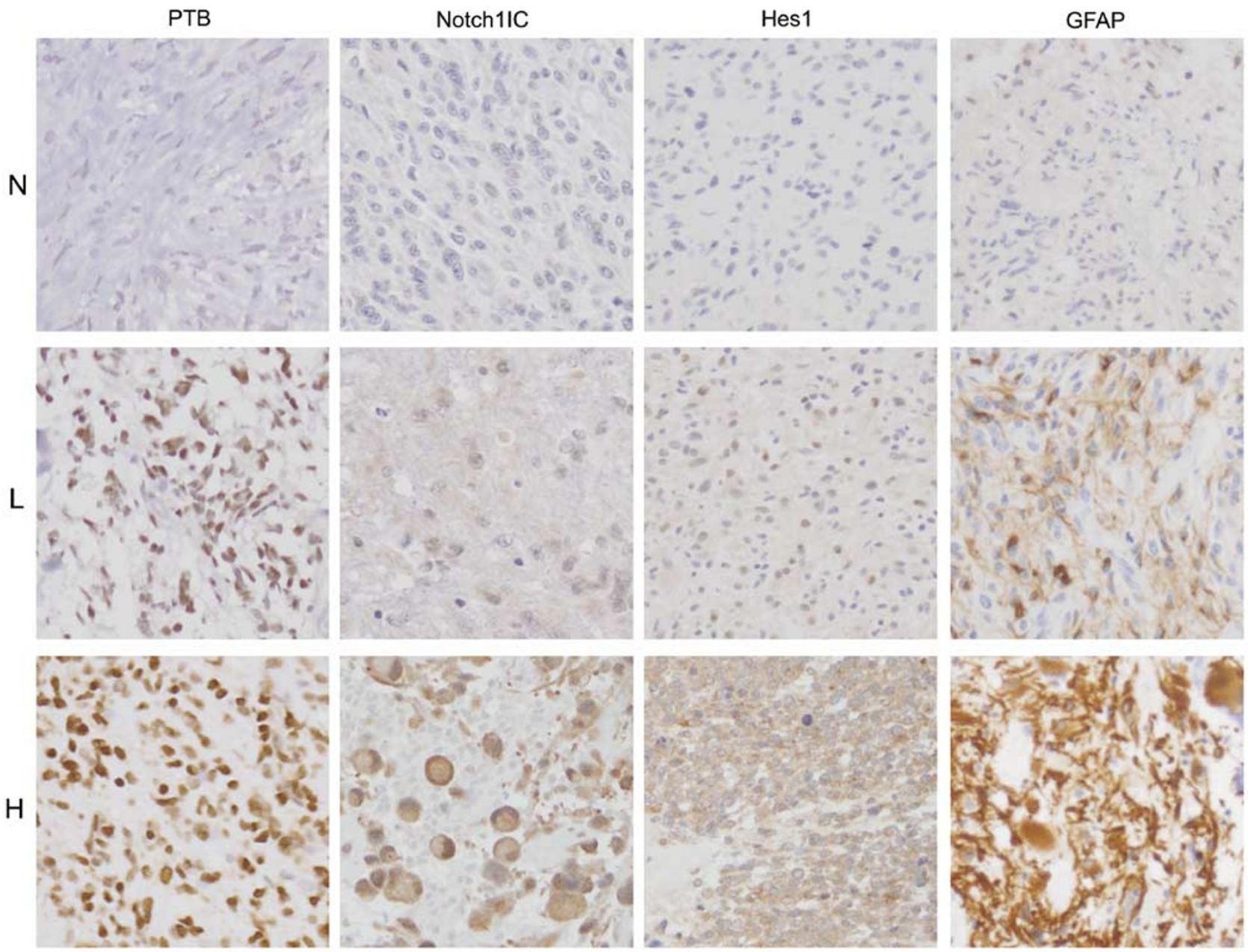

Figure 1 Representative immunohistochemical staining of tissue microarray cores. The upper panel shows normal brain staining of PTB, Notch1IC, Hes1, and GFAP. The predominant staining intensity was Negative for PTB, Negative for Notch1IC, Low Positive for Hes1, and Low Positive for GFAP. The lower panel provides examples of glioblastoma tissue cores scored as Negative (N), Low Positive (L), and High Positive (H) (see Materials and methods). Photomicrographs were taken with a Nikon Microphot-FXA microscope equipped with an Olympus DP70 digital camera.

subset of grade IV tumors (43\%) was Negative for Notch1IC.

To enhance our ability to monitor Notch1 activity, we included staining for the Notch1 target, Hes1. While a greater proportion of glioma samples stained positive for Hes1 compared with normal brain samples, this trend was not statistically significant $(P=0.246)$. Furthermore, there was no observable difference in Hes1 expression in grade IV tumors. Therefore, despite the obvious increase in 

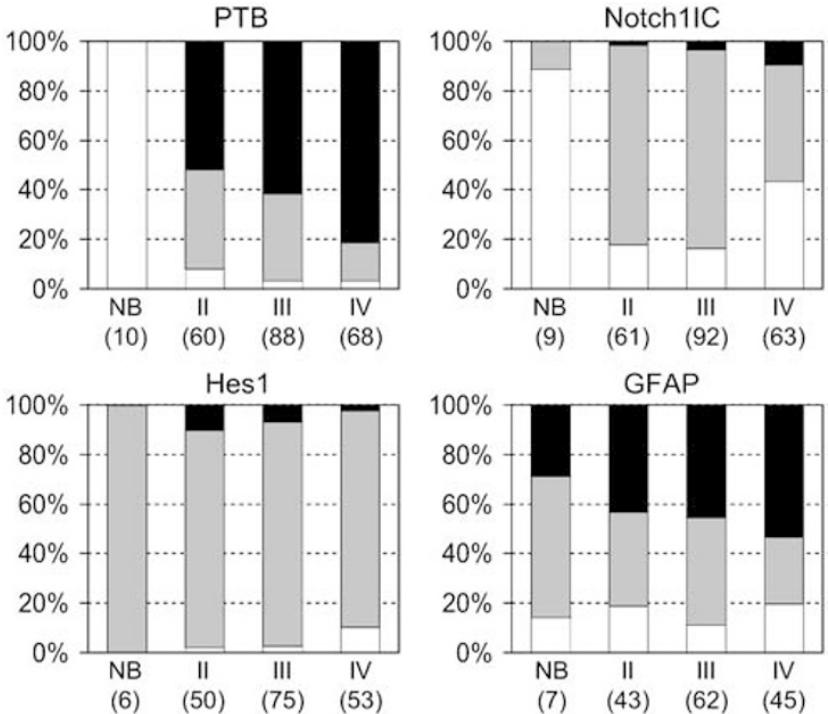

Figure 2 Analysis of tissue microarray staining. Each graph provides the distribution of tissue cores scored as Negative (white), Low Positive (gray), or High Positive (black) for the antibody indicated (see Materials and methods). Samples were grouped according to tissue type: normal brain (NB) and glioma grades II through IV, with the total number of cores used for analysis indicated in parentheses.

Notch1IC levels, the expected concurrent response of Hes1 expression was not observed.

\section{PTB and Notch1IC Expression in Astrocytic and Oligodendrocytic Tumors}

Glial progenitor cells undergo bilateral terminal differentiation into morphologically distinct astrocytes or oligodendrocytes. The cell fate choice can be manipulated by expressing Notch1 in progenitors to drive astrocytic differentiation and inhibit the default oligodendrocyte pathway. ${ }^{17-19}$ We examined PTB and Notch1IC levels in these tumor subtypes (Figure 3). No significant differences in PTB expression existed between the two, but there was a trend towards higher levels of PTB in oligodendrocytic tumors. For Notch1IC, there was a significantly higher number of positive staining in grade III anaplastic oligodendromas than anaplastic astrocytomas $(P=0.03)$, suggesting a greater role for Notch1IC in oligodendromas than in astrocytomas.

\section{PTB Levels were Not Associated with Notch1IC}

In Drosophila, the absence of PTB increases Notch activity. Therefore, we anticipated that tissue samples with low PTB would have high Notch1IC and that the presence of PTB would inhibit Notch activity. The overall trends shown in Figure 2 suggest were that with progressing glioma grade, PTB expression increased, and in a subset of gliomas, Notch1IC decreased. A definitive inverse association between PTB and Notch1IC expression
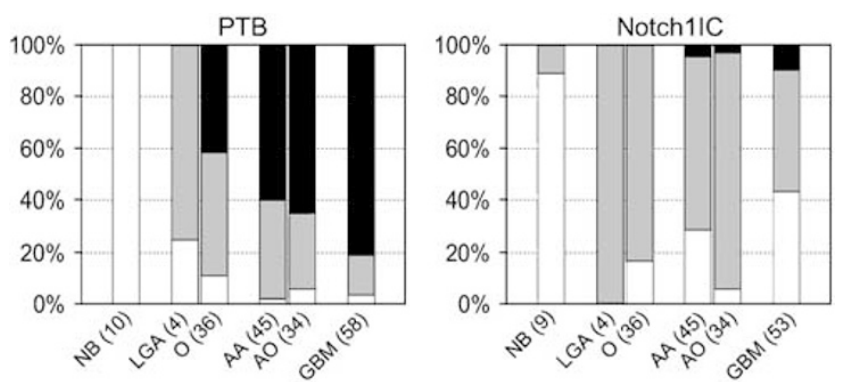

Figure 3 Distribution of PTB and Notch1IC staining in astrocytic and oligodendroglial tumors. The graphs show the distribution of staining intensity for the following tissues: normal brain (NB), low-grade astrocytoma (LGA), oligodendroma (O), anaplastic astrocytoma (AA), anaplastic oligodendroma (AO), and glioblastoma (GBM). The number of samples used for analysis is indicated in parentheses. Tissue cores were scored negative (white), Low Positive (gray), or High Positive (black) as in Figure 2 .
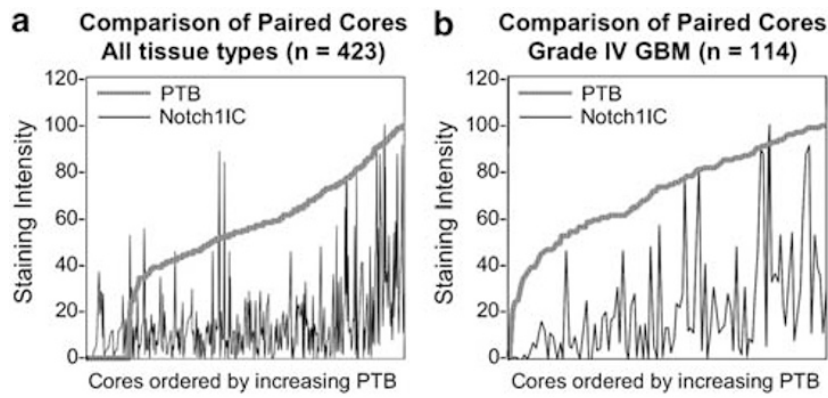

Figure 4 Comparison of PTB and Notch1IC staining intensities in paired serial cores. (a) The graph shows PTB and Notch1IC staining intensity for 423 paired tissue cores. (b) Staining intensity data from the subset of 114 serial cores derived from patients with grade IV glioblastoma. The numeric values for PTB and Notch1IC staining intensities were determined from the scanning analysis (see Materials and methods). Samples are ordered by increasing PTB staining intensity independent of tumor grade.

was not observed, but it was unclear if such a relationship might exist in a subset of gliomas. Therefore, we directly compared PTB and Notch1IC expression for all available paired serial cores, independent of tumor grade (423 core pairs). For this analysis, staining intensity was defined as the sum of the medium and high intensity scores (see Materials and methods). Figure 4a shows a plot of staining intensity for individual paired cores that directly compare PTB and Notch1IC staining. It was immediately obvious that Notch1IC levels were not inversely related to PTB expression levels. A closer examination of expression in 114 grade IV gliomas (Figure 4b), where the trend might be strongest, confirmed that there was no inverse relationship.

\section{Discussion}

The aberrant expression of developmental markers in brain tumors suggests that these tumors revert to 
or arise from undifferentiated cell types. The precise biological role of high PTB levels in normal brain development and gliomagenesis is yet to be determined. While PTB targets several regulators of cell growth, only Notch has been clearly implicated in both neural development and tumorigenesis..$^{9,11,31}$ An unexpected link between Notch activity and PTB expression was first observed in Drosophila, which suggested that PTB functions to attenuate Notch signaling. ${ }^{16}$ As PTB is expressed in gliomas at levels comparable to those in neonates, this provided an opportunity to determine whether Notch activity is inhibited in these tumors. Therefore, we used a large glioma tissue microarray to survey levels of PTB, activated Notch1 (Notch1IC), and Hes1 (a Notch1 target).

The expectation was that Notch1IC would be reduced or absent in tumors expressing high levels of PTB. Staining for PTB revealed variable low levels of expression in normal astrocytes, a finding consistent with our previous results. ${ }^{14}$ There was a significant increase in PTB expression beginning with low-grade tumors $(P<0.001)$, suggesting that upregulation of PTB is an early event during glioma formation. A further enhancement of PTB expression was observed with progressive tumor grade. GBMs had the greatest proportion of samples with high positive staining $(P<0.001)$ compared with other tumor grades. Therefore, GBMs as a group would be predicted to have the lowest frequency of Notch1IC staining. Indeed, a subset of these tumors had a clear absence of Notch1IC expression (Figure 2). However, this group proved to be the exception as there was obvious activation of Notch1 in more than $80 \%$ of grade II and III tumors and most grade IV tumors. Furthermore, a direct comparison of PTB and Notch1IC levels in over 400 paired serial cores definitely demonstrated that an inverse relationship did not exist. Intense Notch1IC staining was found independent of PTB levels, and the lack of Notch1IC in grade IV tumors was not concordant with high levels of PTB (Figure 3). Therefore, it seems unlikely that PTB functions to regulate Notch1 activity in gliomagenesis. Our results do not rule out the possibility that PTB alters Notch activity in other cell types or that glioma cells have developed a mechanism to escape PTB control during tumor progression. In Drosophila, PTB and Notch are related through the fringe glycosylase pathway. A partial rescue of the fringe (fngD4) wing mutant phenotype is found in heph-fringe double mutants. ${ }^{16}$ Because the mammalian genome contains four Notch and four Fringe homologs, one of these genes may be the mammalian target of PTB inhibition and Notch1 may be upregulated as a compensatory response. Whether PTB interacts differentially with any of the mammalian Notch or Fringe homologs remains to be formally examined. Our immunostaining for Hes1 partially addresses this issue because all Notch family members enhance Hes1 expression to varying degrees. ${ }^{31-33}$
Hes1 staining was scored predominantly as Low Positive in normal brain and all glioma grades. A small proportion of gliomas had High Positive Hes1 staining, but this was not statistically significant. A direct comparison of serial sections found no association between Hes1 levels and either Notch1 or PTB levels (data not shown). These findings indirectly rule out PTB-regulated changes in total Notch family member activation and suggest that the role of Notch1IC in gliomagenesis occurs independently of Hes1 targets, perhaps through other targets such as CyclinD1, p21, and NF- $\kappa$ B. ${ }^{25}$

Even though we did not find a relationship between Notch1 regulation and PTB expression, the results of other recent studies suggest that Notch1 independently contributes to gliomagenesis. Purow et $a l^{9}$ found elevated expression of Notch1 and its ligands Delta-like-1 and Jagged-1 in glioma cell lines and human gliomas, and further demonstrated that siRNA knockdown of any of these genes slowed glioma cell growth. Our studies confirm their Notch1 expression findings in a larger patient sample set using an antibody against the active intracellular receptor (Figure 2). Activated Notch was observed in astrocytic tumors, with a trend towards slightly higher levels in oligodendrocytederived tumors (Figure 3). Because the absence of Notch activity promotes oligodendrocyte formation, this finding suggests that these tumors derive from a common progenitor cell type. Our results differ in that we observed a higher percentage of grade IV tumors expressing Notch1: $57 \%$ of 63 samples compared with their $15 \%$ of 20 samples. ${ }^{9}$ In fact, we found that GBMs had the greatest percentage of tumors with High Positive staining (9\%). This discrepancy may simply reflect differences in the total number of patient samples or the sensitivity of the antibodies used. We found inconsistent staining for the total Notch1 antibody; therefore, we chose not to include those data in our analysis (data not shown). Because Notch1 has opposing roles depending on cell type or differentiation state, the subset of grade IV tumors without Notch1IC may behave differently than those with elevated Notch1IC. We are currently expanding our studies to determine whether these differences could have prognostic value.

Our survey of glioma and normal brain tissue samples showed that the development-restricted genes PTB and Notch1 are re-activated in gliomas. Through direct comparisons, we determined that the increase in PTB with glioma grade was unrelated to Notch1IC levels. Grade IV tumors had a pronounced increase in PTB compared with lower glioma grades, whereas only a subset of these tumors had increased Notch1IC expression. Although PTB and Notch are related in Drosophila, they appear to act independently of each other in gliomas. Because PTB is expressed in all tumor grades, it appears to be a critical component of gliomagenesis. In addition to Notch, several other targets of PTB action have 
implicated tumorigenic functions, such as FGFR1, FGFR2, SRC, CLTB, VEGF, APAF1, CASP2, FCGR1A, and $F A S .^{34-42}$ These targets could individually or synergistically provide a growth advantage to glioma cells. This remains to be formally investigated. Unlike PTB, we found a significant loss of Notch1IC expression in a subgroup of grade IV tumors. The presence of Notch may contribute to glioma growth through its ability to renew progenitor cells. If so, aberrant Notch expression may be an indicator of patient outcome and its absence may serve to delineate differences in the aggressiveness of grade IV tumors.

\section{Acknowledgement}

We thank Dr Susan Berget (Baylor College of Medicine, Houston, TX) for providing the DH3 clone of anti-PTB antibody. This work was supported by Grant NIH/NCI CA067946 to GJC. The MD Anderson tissue microarray core lab and LJC were funded by a grant from the Goodwin Foundation.

\section{References}

1 Zhang W, Fuller GN (eds). Genomic and Molecular Neuro-Oncology. Jones and Bartlett Publishers: Sudbury, 2004.

2 Ohgaki H, Kleihues P. Population-based studies on incidence, survival rates, and genetic alterations in astrocytic and oligodendroglial gliomas. J Neuropathol Exp Neurol 2005;64:479-489.

3 Reya T, Morrison SJ, Clarke MF, et al. Stem cells, cancer, and cancer stem cells. Nature 2001;414:105-111.

4 Singh SK, Clarke ID, Terasaki M, et al. Identification of a cancer stem cell in human brain tumors. Cancer Res 2003;63:5821-5828.

5 Sanai N, Alvarez-Buylla A, Berger MS. Neural stem cells and the origin of gliomas. $N$ Engl J Med 2005;353:811-822.

6 Dahlstrand J, Collins VP, Lendahl U. Expression of the class VI intermediate filament nestin in human central nervous system tumors. Cancer Res 1992;52: 5334-5341.

7 Tohyama T, Lee VM, Rorke LB, et al. Nestin expression in embryonic human neuroepithelium and in human neuroepithelial tumor cells. Lab Invest 1992;66: 303-313.

8 Golestaneh N, Mishra B. TGF-beta, neuronal stem cells and glioblastoma. Oncogene 2005;24:5722-5730.

9 Purow BW, Haque RM, Noel MW, et al. Expression of Notch-1 and its ligands, Delta-like-1 and Jagged-1, is critical for glioma cell survival and proliferation. Cancer Res 2005;65:2353-2363.

10 Fomchenko EI, Holland EC. Stem cells and brain cancer. Exp Cell Res 2005;306:323-329.

11 Wagner EJ, Garcia-Blanco MA. Polypyrimidine tract binding protein antagonizes exon definition. Mol Cell Biol 2001;21:3281-3288.

12 Rustighi A, Tessari MA, Vascotto F, et al. A polypyrimidine/polypurine tract within the Hmga2 minimal promoter: a common feature of many growth-related genes. Biochemistry 2002;41:1229-1240.
13 Lillevali K, Kulla A, Ord T. Comparative expression analysis of the genes encoding polypyrimidine tract binding protein (PTB) and its neural homologue (brPTB) in prenatal and postnatal mouse brain. Mech Dev 2001;101:217-220.

14 McCutcheon IE, Hentschel SJ, Fuller GN, et al. Expression of the splicing regulator polypyrimidine tract-binding protein in normal and neoplastic brain. Neuro-oncol 2004;6:9-14.

15 Castrillon DH, Gonczy P, Alexander S, et al. Toward a molecular genetic analysis of spermatogenesis in Drosophila melanogaster: characterization of malesterile mutants generated by single $\mathrm{P}$ element mutagenesis. Genetics 1993;135:489-505.

16 Dansereau DA, Lunke MD, Finkielsztein A, et al. Hephaestus encodes a polypyrimidine tract binding protein that regulates Notch signalling during wing development in Drosophila melanogaster. Development 2002;129:5553-5566.

17 Lundkvist J, Lendahl U. Notch and the birth of glial cells. Trends Neurosci 2001;24:492-494.

18 Gaiano N, Fishell G. The role of notch in promoting glial and neural stem cell fates. Annu Rev Neurosci 2002;25:471-490.

19 Grandbarbe L, Bouissac J, Rand M, et al. Delta-Notch signaling controls the generation of neurons/glia from neural stem cells in a stepwise process. Development 2003;130:1391-1402.

20 Joutel A, Tournier-Lasserve E. Notch signalling pathway and human diseases. Semin Cell Dev Biol 1998;9:619-625.

21 Bresnick EH, Chu J, Christensen HM, et al. Linking Notch signaling, chromatin remodeling, and T-cell leukemogenesis. J Cell Biochem Suppl 2000;79(Suppl 35):46-53.

22 Allenspach EJ, Maillard I, Aster JC, et al. Notch signaling in cancer. Cancer Biol Ther 2002;1:466-476.

23 Brennan K, Brown AM. Is there a role for Notch signalling in human breast cancer? Breast Cancer Res 2003;5:69-75.

24 Maillard I, Pear WS. Notch and cancer: best to avoid the ups and downs. Cancer Cell 2003;3:203-205.

25 Nickoloff BJ, Osborne BA, Miele L. Notch signaling as a therapeutic target in cancer: a new approach to the development of cell fate modifying agents. Oncogene 2003;22:6598-6608.

26 Pan $\mathrm{Y}$, Lin $\mathrm{MH}$, Tian $\mathrm{X}$, et al. gamma-secretase functions through Notch signaling to maintain skin appendages but is not required for their patterning or initial morphogenesis. Dev Cell 2004;7:731-743.

27 Weng AP, Aster JC. Multiple niches for Notch in cancer: context is everything. Curr Opin Genet Dev 2004;14:48-54.

28 Zweidler-McKay PA, Pear WS. Notch and T cell malignancy. Semin Cancer Biol 2004;14:329-340.

29 Hallahan AR, Pritchard JI, Hansen S, et al. The SmoA1 mouse model reveals that notch signaling is critical for the growth and survival of sonic hedgehoginduced medulloblastomas. Cancer Res 2004;64: 7794-7800.

30 Grossman JS, Meyer MI, Wang YC, et al. The use of antibodies to the polypyrimidine tract binding protein (PTB) to analyze the protein components that assemble on alternatively spliced pre-mRNAs that use distant branch points. Rna 1998;4:613-625.

31 Yoon K, Gaiano N. Notch signaling in the mammalian central nervous system: insights from mouse mutants. Nat Neurosci 2005;8:709-715. 
32 Shimizu K, Chiba S, Saito T, et al. Functional diversity among Notch1, Notch2, and Notch3 receptors. Biochem Biophys Res Commun 2002;291:775-779.

$33 \mathrm{Wu}$ Y, Liu Y, Levine EM, et al. Hes1 but not Hes5 regulates an astrocyte versus oligodendrocyte fate choice in glial restricted precursors. Dev Dyn 2003;226: 675-689.

34 Chan RC, Black DL. The polypyrimidine tract binding protein binds upstream of neural cell-specific c-src exon N1 to repress the splicing of the intron downstream. Mol Cell Biol 1997;17:4667-4676.

35 Zhang L, Liu W, Grabowski PJ. Coordinate repression of a trio of neuron-specific splicing events by the splicing regulator PTB. Rna 1999;5:117-130.

36 Jin W, McCutcheon IE, Fuller GN, et al. Fibroblast growth factor receptor-1 alpha-exon exclusion and polypyrimidine tract-binding protein in glioblastoma multiforme tumors. Cancer Res 2000;60:1221-1224.

37 Carstens RP, Wagner EJ, Garcia-Blanco MA. An intronic splicing silencer causes skipping of the IIIb exon of fibroblast growth factor receptor 2 through involvement of polypyrimidine tract binding protein. Mol Cell Biol 2000;20:7388-7400.
38 Cote J, Dupuis S, Wu JY. Polypyrimidine track-binding protein binding downstream of caspase-2 alternative exon 9 represses its inclusion. J Biol Chem 2001;276: 8535-8543.

39 Giraud S, Greco A, Brink M, et al. Translation initiation of the insulin-like growth factor I receptor mRNA is mediated by an internal ribosome entry site. J Biol Chem 2001;276:5668-5675.

40 Mitchell SA, Brown EC, Coldwell MJ, et al. Protein factor requirements of the Apaf-1 internal ribosome entry segment: roles of polypyrimidine tract binding protein and upstream of N-ras. Mol Cell Biol 2001;21: 3364-3374.

41 Coles LS, Bartley MA, Bert A, et al. A multi-protein complex containing cold shock domain (Y-box) and polypyrimidine tract binding proteins forms on the vascular endothelial growth factor mRNA. Potential role in mRNA stabilization. Eur J Biochem 2004;271: 648-660.

42 Izquierdo JM, Majos N, Bonnal S, et al. Regulation of Fas alternative splicing by antagonistic effects of TIA-1 and PTB on exon definition. Mol Cell 2005;19: 475-484. 